\title{
Landslide Emergency Monitoring and Early Warning Based on Situation Awareness Sensing
}

\author{
LIU Peng ${ }^{1}$, HUANG Zheng-jun ${ }^{1 *}$, LI Yong ${ }^{2}$, SONG San-hong ${ }^{3}$, YU Guo1,XIE Mo-wen ${ }^{1}$ \\ ${ }^{1}$ School of Civil and Resource Engineering, University of Science \& Technology Beijing, Beijing 100083, China \\ ${ }^{2}$ Beijing iSafety Technology Co. Ltd \\ ${ }^{3}$ Guodian dadu river houziyan hydropower construction Co. Ltd., kangding, Sichuan 626000, China
}

\begin{abstract}
Compared with the traditional landslide monitoring and early warning technology, landslide emergency monitoring and early warning based on situation awareness sensing has the advantages of active sensing, real-time online diagnosis, combination of timing and triggering acquisition and monitoring. Through the comparison and analysis of the engineering application and traditional technology, this paper shows that the system applies the concept of situation awareness to the field of landslide emergency monitoring effectively and accurately. Besides, the vibration and tilt change of the landslide deformation body is obviously earlier than that of displacement, so it is better to monitor the vibration and tilt change of the landslide deformation body than the change of the displacement of the landslide deformation body.
\end{abstract}

\section{Introduction}

China, with a vast territory, has complex terrain and geological conditions and frequent landslides and other natural disasters, which often cause major property damage and casualties. Therefore, timely and accurate monitoring and prediction of landslides is particularly important.

With the development of science and technology and the unremitting efforts of many scientific researchers, many kinds of effective technologies such as deep displacement monitoring, surface displacement monitoring, close-range photogrammetry, $3 \mathrm{~S}$ technology (remote sensing technology, geographic information system, and global positioning system) ${ }^{[1-5]}$ have been formed.

Yang Lingyun ${ }^{[6]}$ et al. invented a steel strip displacement meter to measure the deformation of the tension fissure on the slope; Chen Pengchao ${ }^{[7]}$ et al. designed a sensing system based on fiber grating sensing technology and successfully applied it to a large-scale landslide monitoring; Xie Mowen ${ }^{[8]}$ et al. applied Terrestrial Laser Scanning (TLS) technology to landslide monitoring and achieved good results. Tang Huiming ${ }^{[9]}$ et al. introduced the principle of azimuth angle measurement technology for inclinometers and discussed the deep displacement monitoring technology.

For landslides that have been loosened, they are in a dangerous state, and emergency monitoring is urgently needed. However, traditional monitoring methods are greatly affected by the external environment. There are some problems in the monitoring of emergency disasters:

\footnotetext{
a First Author: LIU Peng, E-mail:1534088973@qq.com

Corresponding Author: HUANG Zheng-jun, E-mail:huang_jun.0518@163.com
}

(1) The installation is complex, the external work load is large, and it easily causes disturbance to the slope;

(2) Most of them require an external municipal power supply system;

(3) It is impossible to achieve all-day uninterrupted online monitoring;

(4) Data processing is cumbersome and cannot reflect the state of the slope in time.

The microchip sensor monitoring system based on situation awareness can effectively solve the above problems. Based on the situation awareness principle, the system actively monitors the subtle changes of the landslide, which uses the key technologies such as big data to analyze the landslide deformation status and evolution trend in real time and determines the safety status of the landslide body in a timely and effective manner. Besides, it also provides accurate, comprehensive and timely landslide warning information to surrounding residents and management departments through the cloud platform.

In this paper, a microchip sensing technology based on situation awareness is introduced and applied to landslide emergency monitoring and forecasting. As a new technique and method for landslide deformation monitoring, it provides technical support for the emergency monitoring and early warning of landslide disaster and has great application value.

\section{Microchip sensor technology}

2.1Situation awareness and landslide
monitoring


Situation awareness stems ${ }^{[10]}$ from the study of Human Factors in space flight. Endsley [11] defined situation awareness as"the acquisition and understanding of environmental factors and the prediction of future conditions in a certain time and space. After that, it has been widely applied in military battlefields, nuclear reaction control, air traffic supervision and medical emergency dispatch.

At present, situation awareness is mostly used in the field of network security research and is rarely researched in landslide monitoring. Landslide deformation is a dynamic process in a complex environment. The unstable failure of a landslide is a progressive failure process from gradual deformation to sudden failure ${ }^{[12]}$, which can be divided into a creeping landslide and a sudden landslide according to the failure rate. ${ }^{[13]}$

From the initial deformation to the overall sliding failure process, the landslide can be divided into three stages: constant speed deformation, accelerated deformation, and acceleration of critical sliding ${ }^{[14]}$ (Fig. 1 ). If the key parameters are monitored and obtained during the various stages of landslide deformation, a three-level landslide monitoring model [15] (Fig.2) is established according to the situation awareness principle, which can effectively monitor the real-time state of landslide deformation and perceive the future trend.

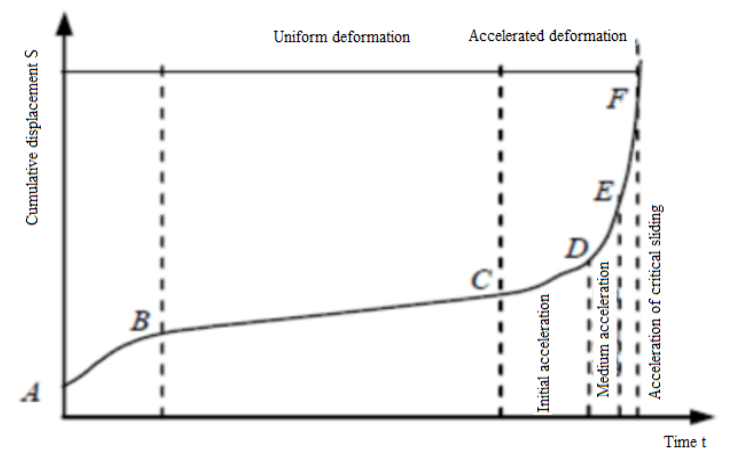

Figure 1. Three stages of landslide deformation

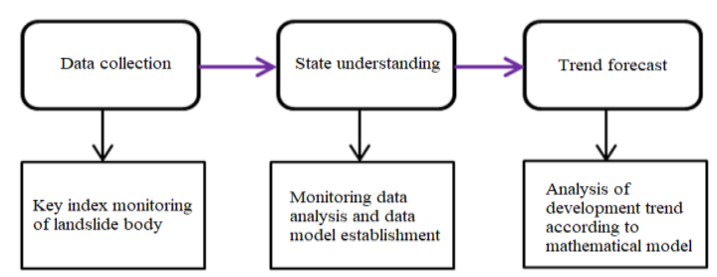

Figure 2. Three-level model of landslide monitoring situation awareness

\subsection{Microchip sensor system composition and advantages}

The microchip sensor system consists of microchip acquisition terminal, microchip station, isafety cloud platform, and mobile phone client (Fig.3). The microchip acquisition terminal has a built-in wireless situation awareness sensor with low power consumption, high sensitivity, and strong durability. It can acquire information such as space deformation, relative deformation, and vibration at regular or threshold values. At the same time, external sensors can be used to collect seepage and osmotic pressure, stress -strain, temperature, real-time positioning and other indicators according to actual needs, to achieve real-time online monitoring of subtle changes in the landslide. The collected index data is wirelessly transmitted to the microchip station through the 485 communication signal; the microchip station then transmits the received signal to the isafety cloud platform through GPRS; the staff can check the status of the landslide body at any time through the mobile phone or the computer. The site monitoring layout is shown in Figure 4.

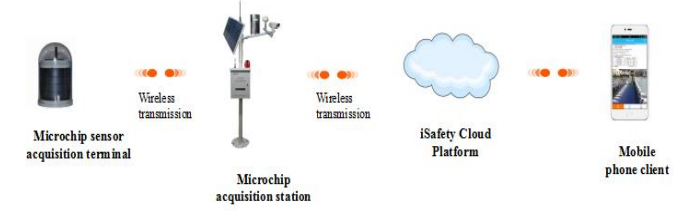

Figure 3. The map of microchip sensor system

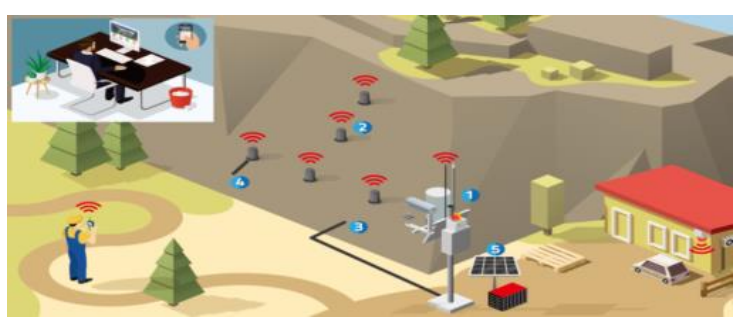

Figure 4. The diagram of field monitoring simulation

The system has the characteristics of wireless transmission, self-power supply, maintenance-free management, and easy installation. Thus, it can quickly and accurately determine the landslide body deformation trend and other forms. Compared with traditional monitoring equipment, the system has the following advantages:

(1) The system can actively sense, automatically analyze, and real-time online diagnose landslide emergency warning information ;

(2) The system is equipped with three independent power supply systems for capacitors, lithium batteries and solar panels, and no external power supply is required ;

(3) The iSafety cloud platform can manage data, quickly calculate, intelligently analyze, and make 
scientific decisions. The staff only needs to access the platform through the mobile phone to perform data query and warning information viewing, and realize accurate, comprehensive, and timely safety warning of landslide deformation monitoring ;

(4)The system has good stability, convenient installation and operation, and no special maintenance;

(5) The data acquisition can collect the index data of any sudden change time at the same time as collecting at regular intervals. In a special period, it can also remotely and manually collect the monitoring index data to realize on-demand encryption monitoring.

\section{Application example}

\subsection{Project Overview}

During the storage period of a reservoir, the inspectors found that there were signs of large deformations on the high retaining wall and the measured slope surface on the outside of the road in the gully section of the open slope at the top of the open slope of the reservoir area, which had seriously jeopardized the traffic safety of provincial roads on the lower slope of the slope (Fig.5 and Fig. 6). The length of the slope body deformation along the direction of the river was about $460 \mathrm{~m}$; the elevation of the slope was $2080 \mathrm{~m}$; the bottom elevation was below $1820 \mathrm{~m}$; the natural slope was $38^{\circ} \sim 46^{\circ}$, and the total deformation volume was about 4 million $\mathrm{m}^{3}$. The top surface of the open top landslide was a gypsum, a massive stone, and a crushed rock with a collapsed layer. The total volume of the solitary stone group was about $150,000 \mathrm{~m}^{3}$. The underlying area was the Silurian greenschist, which contains phyllite, and there was an inter-layer crushing zone in the rock. The surface cracks were mainly distributed in the rear part of the deformed body. In addition, the fractures at the downstream and the trailing edge of the deformed body were basically penetrated, and the arc fracture had formed locally. Indeed, the ruptured landslide body was in an extremely dangerous state and safety monitoring and early warning were required immediately.

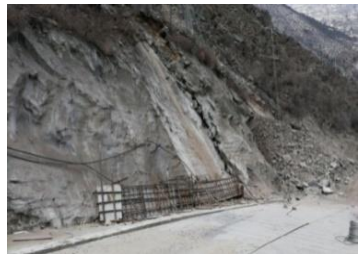

Figure 5. On-site slope rockfall

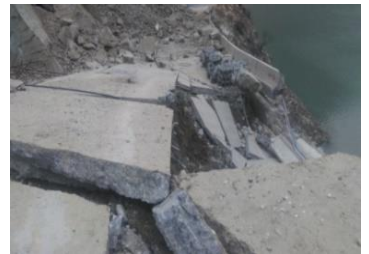

Figure 6. On-site damaged section

\subsection{Monitoring scheme}

According to the site conditions and the requirements of the management department, as well as through expert consultation and demonstration, it was decided to use the microchip sensor monitoring system to carry out safety monitoring on the deformation of the open top slope landslide, and the total station was used as the comparison monitoring method.

The monitoring scheme was to conduct continuous on-line monitoring of the vibration acceleration, inclination, dip angle change and velocity at different locations on the open slope landslide. Through data analysis, the law of tilt, vibration and displacement change of the monitoring point are provided. Besides, the real-time status and change trend of landslide deformation were effectively monitored and forecasted in conjunction with the displacement data of the total station measuring points.

The monitoring scheme had a total of 7 microchip monitoring points (W1-W7) and 1 microchip station, of which monitoring points 1 to 3,6 and 7 were arranged on the slope, and monitoring points 4 and 5 were arranged on the foot of the mountain (Fig. 7). The collection interval was set to 1 hour for timing collection and different monitoring sites were set with $2^{\circ \sim 5^{\circ}}$ and $1 \mathrm{~g}^{\sim} 2 \mathrm{~g}$ yellow warning and red warning thresholds. At the same time, 15 total station TP monitoring points were arranged on site. The No. 1, No. 2, No. 4 and No. 6 microchip monitoring points and the No. 4 , No. 3 , No. 8 , No. 10 and No. 12 total station TP monitoring points were basically the same, and the interactions were compared and analyzed (Fig. 8).

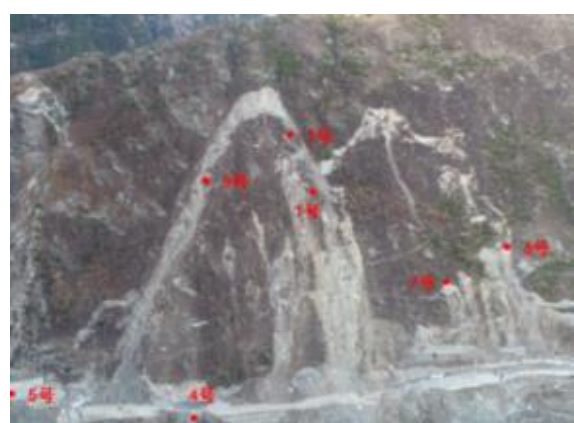

Figure 7. The layout of monitoring points

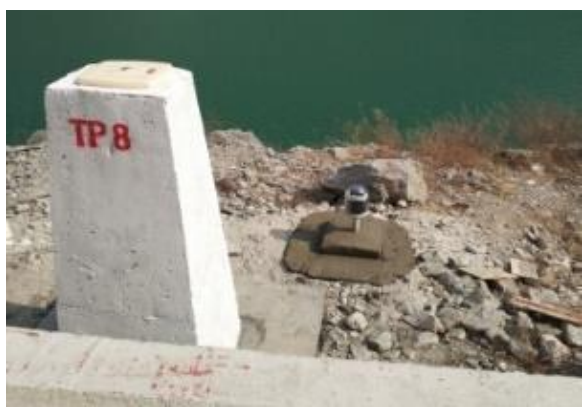


Figure 8. The monitoring points of TP8 and W2

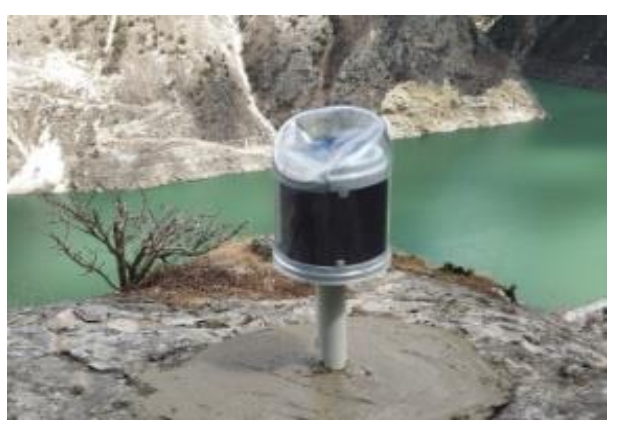

Figure 9. No. 3 collection terminal

\subsection{Monitoring data analysis}

During the period from January 6, 2018 to March 23, 2018, more than 100 cracks had been produced, with a crack width of 5 to $80 \mathrm{~cm}$, a length of 30 to $60 \mathrm{~m}$, and a maximum crack misalignment of $70 \mathrm{~cm}$. In the landslide area, a total of more than 300 tilt angles and vibration alert values were triggered, and the risk aversion was successfully performed 8 times. The comprehensive process curve of the landslide deformation body is shown in Figure 10.

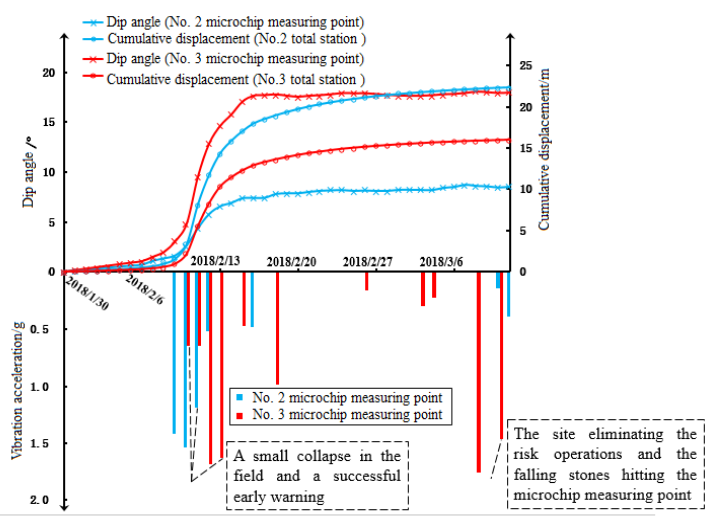

Figure 10. The comprehensive process curve of the landslide deformation body

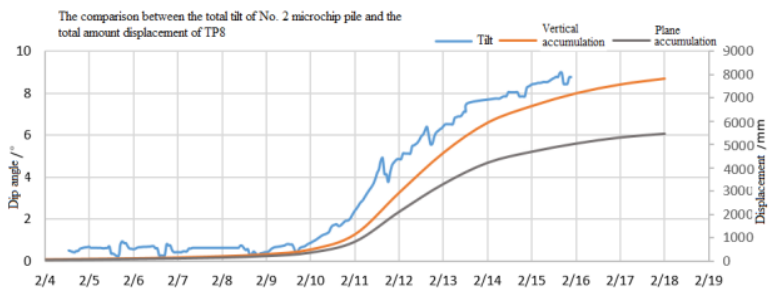

Figure 11. The comparison curve between the total tilt of W2 and the total amount displacement of TP8

Due to space limitations, the daily monitoring data of No. 2 microchip monitoring point and the daily monitoring data of the total station TP8 monitoring point were taken as an example for com parison and analysis. The linear comparison between the total tilt of No. 2 microchip monitoring point and the total displacement process of TP8 is shown in Fig. 11. Similarly, the comparison between the tilt daily increment of No. 2 microchip monitoring point and the displacement daily increment of TP8 is shown in Fig. 13.

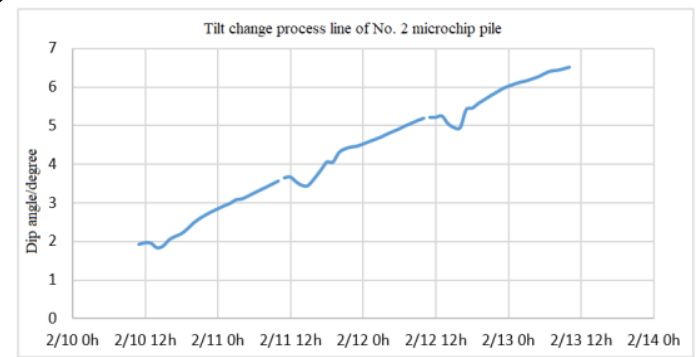

Figure 12. Tilt change process line of $\mathrm{W} 2$

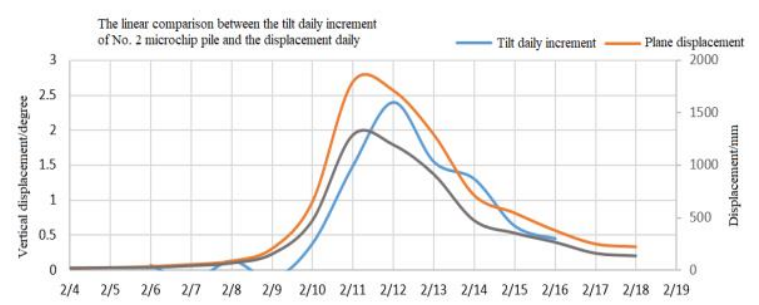

Figure 13. The linear comparison between the tilt daily increment of the No. 2 microchip monitoring point and the displacement daily increment of TP8

From the figure, it can be seen that from January 16 to January 24 , the daily variation of the tilt angle measured by the microchip collection terminal gradually increases. From the 10th of February, the daily variation of the tilt angle measured by the microchip collection terminal was significantly increased (Fig.12). After February 23rd, the deformation of the landslide body became stable; the site began to eliminate the risk operations on the dangerous rock on the slope. On February 26th, the falling of the dangerous rock in the No. 3 microchip collection terminal caused by eliminate the risk operations (as shown in Fig. 9) was issued in a timely manner.

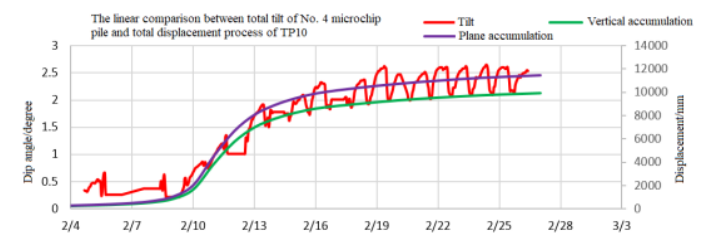

Figure 14. The linear comparison between total tilt of W4 and total displacement of TP10

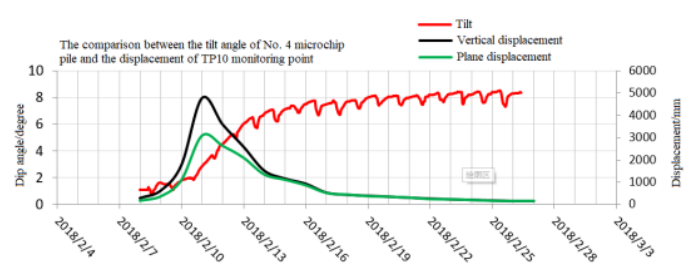


Figure 15. The comparison between the tilt angle of W4 and the monitoring point displacement of TP10

It can be seen from Fig. 14 that the variation rule of the total tilt of $\mathrm{W} 4$ and the change rule of the total amount of displacement detected by the TP10 monitoring point are in good agreement. From February 10 to 11 , the data can be analyzed: whether it is plane displacement or vertical displacement, the change of dip angle is earlier than the change of displacement. Therefore, the occurrence of deformation displacement of the slope body can be well predicted based on the change trend of the inclination angle. In Fig.15, the linear comparison between the tilt angle of W4 and the monitoring point displacement of TP10 can further verify that the change of the deformation of the slope body can be predicted based on the monitoring of the change trend of its tilt angle. Moreover, the forecast effect is even more pronounced, especially when the daily deformation reaches more than a hundred millimeters.

\section{Conclusion}

Compared with the traditional landslide monitoring and early warning technology, landslide emergency monitoring and early warning based on situation awareness sensing has the advantages of active, comprehensive, timely, online monitoring and so on. Moreover, through comparative analysis of practical engineering applications, the following conclusions are drawn:

(1) The microchip sensor system is a new landslide monitoring and early warning technology based on the concept of situation awareness of "environmental factors acquisition and future state prediction"[16], especially for landslide emergency early warning monitoring. The system's advantages of active sensing, real-time monitoring, automatic wireless monitoring, low power consumption, maintenance-free, etc. can be fully reflected and effectively applied.

(2) By comparing with the deformation displacement and inclination angle of the landslide body, we can predict by monitoring the trend of inclination angle. The monitoring effect is better, especially when the deformation amount reaches several tens of millimeters a day.

( 3 ) The dramatic changes in landslide deformation displacement first bring about changes in vibration and tilt, and vibration and tilt change significantly earlier than the change in displacement. Therefore, it is possible to monitor and predict the deformation of landslides through real-time and threshold-valued triggering of vibration and tilt, while the effect of early warning is significantly better than that of displacement monitoring.

\section{References}

1. FAN Yongbo,HOU Yuefeng,et al. Landslide stability analysis based on surface and Deep Displacement Monitoring data [J]. Journal of EuRiueeriuR- GeoLoRy,2013,21(6): 885-891

2. CAI Jia-lun,ZHANG Wen-jun,et al. Landslide stability analysis based on law of surface displacement[J]. Science of Surveying and Mapping.2016.3(3):96-99

3. YNG Hua-chao,DENG Ka-zhong,et al. Monitoring technique for deformation measurement of similar material model with digital close-ange photogrammetry[J]. Journal of China Coal Society, 2006.6(3): 292-295

4. LI Yonghsu. Research on the Remote Sensing Dynamic character monitoring and prediction analysis of Landslide Disaster[D]. CHENGDU Southwest Jiaotong University. 2017,1

5. WANG Chunxiang, JIANG Yujing, XIE Mowen,et al. Prediction and Assessment of regional Slopefailure Hazard based on gis[J]. Chinese Journal of Rock Mechanics and Engineering. 2008,12(12)

6. YANG Ling-yun. A Simple and Practical Landslide Displacement Monitoring Technology --- Steel tube bamboo strip displacement meter method [J]. SWCC 2003(10):37-38

7. CHEN Peng-chao,LI Jun, et al. Monitoring technology of pipelines using fiber bragg grating and its application in landslide areas[J]. Chinese Journal of Geotechnical Engineering, 2010.32(6): 897-902

8. Xie M W,Hu M,Du Y,et al. Application of TLS technique to landslide monitoring: Summarization and prospect $[\mathrm{J}]$. Remote Sensing for Land and Resources. 2014,26( 3):8-15

9. TANG Huiming,CAI Yi,et al. Current Status and Prospect for Inclinometer Application in Monitoring of Landslide Deep Displacement [J]. Journal of Engineering Geology. 2016.s1.102 702-709

10. Theureau J. Use of nuclear reactor control room simulators inre search \&. development. In: 7th IFAC/IFORS/IEA Symposium on Analysis, Design and Evaluation of MAN MACHINE SYSTEMS, Kyoto. 1998. 425-430

11. Endsley M R. Design and evaluation for situation awareness enhancement. Paper presented at the Human Factors Society 32nd Annual Meeting. Santa Monica, CA, 1988

12. ZHENG Lining. Research of failure mechanism and the local failure zones for Consequent Slope based on strain softening theory [D]. Southwest Jiaotong University, 2012

13. LIU Guangrun, YAN Echuan LIAN Cao. Discussion on Classification of Landslides [J]. Journal of Engineering,2002,10(4): 340-342 
14. XIAO Jin. Theory and Practice of the treatment of Vital Landslide Hazard [D]. Chengdu University of Technology,2009

15. WANG Hug Qiang, LAI Ji Bao, ZH U Liang,et al. Survey of Network Situation Awareness System [J]. Computer Science, 2006,5(10)

16. YANG Song, YANG Qiang, YANG Zhao-Hui,et al. Battlefield Situation-ware Interface Model on Context [J]. Command Control \& Simulation,2017,4(2):82-84 